\title{
Gradual eddy-wave crossover in superfluid turbulence.
}

\author{
Victor S. L’vov* ${ }^{*}$, Sergey V. Nazarenko ${ }^{\dagger}$ and Oleksii Rudenko* \\ *Department of Chemical Physics, The Weizmann Institute of Science, Rehovot 76100, Israel \\ ${ }^{\dagger}$ University of Warwick, Mathematics Institute, Coventry, CV4 7AL, UK and \\ ¥ Department of Theoretical Physics, Institute for Magnetism, Ukraine National Ac. of Sci., Kiev, Ukraine
}

\begin{abstract}
We revise the theory of superfluid turbulence near the absolute zero of temperature and suggest a model with differential approximation for the energy fluxes in the $k$-space, $\varepsilon_{\mathrm{HD}}(k)$ and $\varepsilon_{\mathrm{KW}}(k)$, carried, respectively, by the collective hydrodynamic (HD) motions of quantized vortex lines and by their individual uncorrelated motions known as Kelvin waves (KW). The model predicts energy spectra of the HD and the KW components of the system, $\mathcal{E}_{\mathrm{HD}}(k)$ and $\mathcal{E}_{\mathrm{KW}}(k)$, which experience a smooth crossover between different regimes of motion over a finite range of scales. For an experimentally relevant range of $\Lambda \equiv \ln (\ell / a)$ ( $\ell$ is the mean intervortex separation and $a$ is the vortex core radius) between 10 and 30 the total energy flux $\varepsilon=\varepsilon_{\mathrm{HD}}(k)+\varepsilon_{\mathrm{KW}}(k)$ and the total energy spectrum $\mathcal{E}(k)=\mathcal{E}_{\mathrm{HD}}(k)+\mathcal{E}_{\mathrm{KW}}(k)$ are dominated by the HD motions for $k \lesssim 2 / \ell$. In this region $\mathcal{E}(k)$ follows the HD spectrum with constant energy flux $\varepsilon \simeq \varepsilon_{\mathrm{HD}}=$ const.: $\mathcal{E}(k) \propto k^{-5 / 3}$ for smaller $k$ and tends to equipartition of the HD energy $\mathcal{E}(k) \propto k^{2}$ for larger $k$. This bottleneck accumulation of the energy spectrum is milder than the one predicted before in [19] based on a model with sharp HD-KW transition. For $\Lambda=30$, it results in prediction for the effective viscosity $\nu^{\prime} \simeq 0.002 \kappa(\kappa$ is the circulation quantum) which is in a reasonable agreement with its experimental value in ${ }^{4} \mathrm{He}$ low-temperature experiment $\approx 0.003 \kappa$. For $k \gtrsim 2 / \ell$, the energy spectrum is dominated by the KW component: almost flux-less $\mathrm{KW}$ component close to the thermodynamic equilibrium, $\mathcal{E} \approx \mathcal{E}_{\mathrm{KW}} \approx$ const at smaller $k$ and the $\mathrm{KW}$ cascade spectrum $\mathcal{E}(k) \rightarrow \mathcal{E}_{\mathrm{KW}}(k) \propto k^{-7 / 5}$ at larger $k$.
\end{abstract}

\section{INTRODUCTION}

Liquid ${ }^{4} \mathrm{He}$ and ${ }^{3} \mathrm{He}$ at very low temperatures can be viewed as a perfect superfluid without any normal fluid present. Turbulence is a very common state for such superfluids, and it comprises one of the most interesting subjects in physics with exciting recent developments in turbulence; see, e.g. [1-16]. Superfluid turbulence consists of a tangle of quantized vortex lines $[1-4,17,18]$. How is superfluid turbulence related to usual hydrodynamical turbulence? On the one hand, at the scales greater than the mean distance between the inter-vortex separation distance one can expect the vortex discreteness to be unimportant and, therefore, superfluid and hydrodynamical turbulence should have similar properties at these scales. This can be true, of course, if the superfluid vortex tangle is not completely random but polarized and organized into vortex bundles which at large scales form similar motions as would continuous hydrodynamic eddies. In turn, such a vortex polarization can be either introduced by the external forcing (yet to be understood how), or it can occur due to a (yet to be found) self-organization mechanism. On the other hand, since there is no viscosity, the energy would cascade in superfluid turbulence downscale without loss until it reaches to the small scales where the quantum discreteness of vorticity is important. It is believed that at this point the Kolmogorov-type (K41) eddy dominated cascade is replaced by a cascade due to nonlinearly interacting Kelvin waves. Kelvin wave cascade takes energy further downscale where it can be radiated away by phonons.

Although the overall picture of superfluid turbulence described above seems quite reasonable, some important details of this picture are yet to be established. A par- ticularly interesting question is about the structure of the crossover between the eddy dominated and the wave dominated regions of the spectrum. As it was pointed out in our recent paper [19], the nonlinear transfer mechanisms among weakly nonlinear Kelvin waves on discrete vortex lines is less efficient than the energy transfers due to the strongly nonlinear eddy-eddy interactions in continuous fluids. This results is an energy cascade stagnation at the crossover from the collective eddy dominated to the single-vortex wave dominated scales. The main message of paper [19] is that such a bottleneck phenomenon is robust and common for all the situations where the energy cascades experiences a continuous-todiscrete transition, and the details of particular mechanism of this transition are secondary. Indeed, most discrete physical processes are less efficient than their continuous counterparts [26]. On the other hand, particular mechanisms of the continuous-to-discrete transition can obviously lead to different strengths of the bottleneck. The quantitative measure of the bottleneck is the value of rms vorticity, because this is the quantity which enters into the definition of the effective viscosity which is experimentally observable via measuring decay of the vortex line density $[1,7]$ (see below).

Paper [19] considers the bottleneck mechanism under the simplest assumption that a sharp transition from the K41 eddy dominated cascade to the Kelvin wave weak turbulence occurs at the mean inter-vortex separation scale $\ell$. In this case, the vortex line reconnections provide a mechanism to transfer the energy from the eddy to the wave motions, but their role for the energy cascade itself was neglected (even though it was noted that the bottleneck strength can be affected if this role was taken into account). On the other hand, paper [20] considered 
another extreme when the reconnections are the key process for the crossover cascade which was suggested to go through three different stages in a rather narrow range of scales of width $\Lambda,-$ bundle-dominated, nearest neighbor and self reconnections. In spite of this rather unrealistic construction, the end result was still a bottleneck and reduction of the effective viscosity, though by a smaller factor than predicted in [19], $\Lambda$ instead of $\Lambda^{5}$.

In the present paper we neglect the role of reconnections for the cascade process because, as we argued in [19], the reconnections are strongly inhibited within the polarized vortex bundles, and their occurrence is limited to the edges of these bundles. Since the volume in between of the bundles is small compared to the volume inside of these bundles, it seems natural to assume that the main contribution to the cascade will be due to nonlinear dynamics of non-reconnecting vortex lines inside the vortex bundles, even though it is still possible that the reconnections can adjust the strength of the bottleneck, particularly if the K41 range is not too large and the turbulence polarization is reduced. The final answer about the role of reconnections should, of course, be sought in experimental and numerical data. In the present paper, we extend the analysis of [19] by taking into account the fact that Kelvin waves can be generated and play a role in the energy cascade at the scales greater than $\ell$, and that the transition from the eddy to the wave motions occurs over an extended range of scales rather than sharply.

\section{BOTTLENECK SCENARIO WITH SHARP CROSSOVERS}

\section{A. Dimensional- and velocity-crossover scales}

When the "bottleneck" effect was first described in [19], it was assumed that the crossover from the eddy motions to the wave motions happens sharply at the scale $\simeq \ell$, i.e. mean intervortex distance, or in the $k$-space at $k \simeq k_{\text {dim }}$, where

$$
k_{\mathrm{dim}} \ell \simeq 1 .
$$

Subscript " dim" reminds that estimate (1a) follows from the simplest possible dimensional reasoning. Besides, it roughly corresponds to an idea that at the scales larger than $\ell$ the vortex lines must be polarized and form bundles which would correspond, in a course grained sense, usual hydrodynamic eddies, while for $k>k_{\text {dim }}$ the vortex motions can be viewed as oscillations independently happening on individual vortex lines, i.e. as 1D Kelvin waves. On the other hand, it was also remarked in [19] that the self-induced motion of the vortex line can get faster than its motion due to the collective interaction with the other vortices in the bundle already at the scales $k \gtrsim k_{\mathrm{vel}}$, where

$$
k_{\mathrm{vel}} \ell \simeq \sqrt{2 / \Lambda}
$$

Subscript " vel " reminds that estimate (1b) follows from comparison of the self-induced velocity with crossvelocity induced at a given vortex line by nearby $\ell$-distant vortex line. A more detailed discussion of this characteristic scale, which clarifies factor " 2 " under the square root, is given below in Appendix A 2. Thus, Kelvin waves can be expected to be present in some form already in the wave-vector range from $k_{\text {vel }}$ to $k_{\text {dim }}$ where they would coexist with the collective/eddy motions. It was argued, however, that due to their oscillatory character the waves would contribute much less into the cumulative motion of the vortex bundles in this scale range.

Before going into details what occurs in the transition range

$$
k_{\mathrm{vel}} \lesssim k \lesssim k_{\mathrm{dim}}
$$

it is worthwhile to reconsider some aspects of the problem under the simplest assumption that only one crossover scale is relevant. This is the subject of the current section.

\section{B. Bottleneck predictions in the presence of dimensional- and velocity-crossovers}

Here we reconsider the simplest scenarios of the eddywave transition with a sharp crossover with the only difference from [19] that the crossover scale $k_{*}$ is not necessarily at $k_{\mathrm{dim}} \simeq 1 / \ell$ but lies somewhere in the range (1c). To this end, we remind the Kozik-Svistunov [23] spectrum of Kelvin waves, in the form suggested in [19]:

$$
\mathcal{E}_{\mathrm{KW}}(k) \simeq \Lambda\left(\kappa^{7} \varepsilon / \ell^{8}\right)^{1 / 5}|k|^{-7 / 5}
$$

Here $\mathcal{E}_{\mathrm{KW}}(k)$ is the one-dimensional (in the $\boldsymbol{k}$-space) energy density of Kelvin waves, normalized such that $E_{\mathrm{KW}}=\int \mathcal{E}_{\mathrm{KW}}(k) d k$ is their total energy in unite volume, $\kappa$ is the quantum circulation and $\varepsilon$ is the energy flux over scales. Parameters $\varepsilon$ and $\ell$ are mutually dependent, and their relation follows from the expression for the rms vorticity in the system of quantum filaments, $\sqrt{\left\langle|\omega|^{2}\right\rangle} \simeq \kappa \ell^{-2}$. The later can be found for well developed turbulence when $\left\langle|\omega|^{2}\right\rangle$ is dominated by the classical-quantum crossover scale $k_{*}$. In the present case

$$
\langle|\omega|\rangle^{2}=2 \int k^{2} \mathcal{E}_{\mathrm{HD}}(k) d k \simeq k_{*}^{3} \mathcal{E}_{\mathrm{KW}}\left(k_{*}\right),
$$

which, after substitution of (2), gives

$$
\varepsilon \simeq \kappa^{3} / \Lambda^{5} \ell^{12} k_{*}^{8} .
$$

Factor 2 in Eq. (3) follows from summation over vector indexes under assumption of isotropy of turbulent spectra. Equation (4) corresponds to the effective viscosity

$$
\nu^{\prime}=\frac{\varepsilon \ell^{4}}{\kappa^{2}} \simeq \kappa / \Lambda^{5}\left(k_{*} \ell\right)^{8} .
$$


Under the simplest assumption $k_{*} \simeq k_{\mathrm{dim}} \simeq 1 / \ell$ one gets the value, reported in [19]: $\nu^{\prime} \simeq \kappa / \Lambda^{5}$. For the sharp crossover at the velocity-crossover scale $k_{\mathrm{vel}} \simeq 1 /(\ell \sqrt{\Lambda})$, where the self-induced velocity is of the order of the crossinduced velocity (see below), one gets $\nu^{\prime} \simeq \kappa / \Lambda$. As we argued we expect that the true value of the crossover scale is somewhere in the region (1c). Thus one expects that the true value of the bottleneck and corresponding $\nu^{\prime}$ is somewhere in between of the two extreme values

$$
\kappa \Lambda^{-5} \lesssim \nu^{\prime} \lesssim \kappa \Lambda^{-1}
$$

Note that formally the value $\nu^{\prime} \simeq \kappa \Lambda^{-1}$ is the same as the one predicted by the Kozik and Svistunov approach based on reconnections [20].

\section{Bottleneck at sharp amplitude-crossover}

Let us now check the consistency of the assumed in the previous section sharp crossover at some $k_{*}$ in the interval (1c). To this goal let us evaluate the amplitude $h\left(k_{*}\right)$ of the Kelvin waves at this scale. Obviously, for consistency this amplitude must remain less than the intervortex separation $h\left(k_{*}\right) \lesssim \ell$. This allows one to introduce the amplitude-crossover scale $k_{\mathrm{amp}}$, at which

$$
h\left(k_{\mathrm{amp}}\right) \simeq \ell .
$$

The estimate for $h(k)$ can be obtained from the Hamiltonian of Kelvin wave in the so-called local-induction approximation (see, e.g., Eq. (5b) in our Ref. [19]):

$$
h(k) \simeq \sqrt{\mathcal{E}_{\mathrm{KW}}(k) / \Lambda \kappa^{2} k^{3}} .
$$

Substituting here $\mathcal{E}_{\mathrm{KW}}(k)$ from Eq. (2) and using Eqs. (4) and (7a) one gets estimate

$$
k_{\mathrm{amp}} \ell \simeq \sqrt[6]{2 / \Lambda}
$$

which is inside of the region (6). The subscript "amp" reminds that estimate (7c) follows from comparison of the wave amplitude with the intervortex distance. Factor " 2 " under the root is put by analogy with Eq. (1b) to ensure that $k_{\mathrm{vel}}<k_{\mathrm{amp}}$ for any $\Lambda$. Assuming a sharp crossover at this scale one gets from Eq. (5):

$$
\nu^{\prime} \simeq \kappa \Lambda^{-11 / 3},
$$

which is, as expected, within the range (6).

So, in order the waves amplitude to be less than the intervortex distance, the inequality $k>k_{\mathrm{amp}}$ must hold. The problem is to clarify what is going on in the interval

$$
k_{\mathrm{vel}} \lesssim k \lesssim k_{\mathrm{amp}}
$$

where formally computed [with the Kelvin-wave spectrum (2)] wave amplitude $h$ exceeds the intervortex distance $\ell$, which cannot physically happen. For example, at the scale $k_{\mathrm{vel}}$ one gets from Eq. (7b): $h \simeq \Lambda \ell \gg \ell$. On the other hand, the motions with $k>k_{\text {vel }}$ cannot be considered as pure collective, because the cross-velocity (which is the influence of the motion of one vortex line in the place of another one) is smaller than the self-induced velocity (for more detailed discussion of this question, see Appendix A 2).

Our scenario, is that in the interval (9) the growth of the wave amplitude on a particular vortex line would be arrested by the adjacent vortex lines in the bundle which would "get in the way". Speculations of similar type of Kozik and Svistunov [20] lead them to a suggestion that the hydrodynamic and the wave turbulence ranges are separated by the range of scales where the energy cascade is dominated by the vortex line reconnections. On the other hand, it was pointed out in [19] that, because the vortex lines in turbulence must be polarized and organizes in bundles, the reconnection process must be suppressed and pushed to small volumes in between of the vortex bundles. Instead of a reconnection, one can expect a restriction of the wave motion of an individual vortex line when it grows in amplitude and tries to push close to the other vortex lines in the bundle. Naturally the growth of such a wave would get arrested at the amplitude when the inter-vortex energy (which grows due to shortening of the distance to the considered vortex line) becomes equal to the vortex self-energy.

This leads us to the following physical model of turbulence in the range (9). In the $\mathbf{x}$-space, turbulence consists of vortex bundles with a fractal structure. Each vortex bundle which is made of denser sub-bundles, such that the mean separation of lines within the sub-bundle is $\ll \ell$ and the mean distance between the sub-bundles is $\gg \ell$. In turn, each sub-bundle consists of even denser sub-sub-bundles, etc. The density of vortex lines within a particular sub-bundle is such that at the scale of this sub-bundle the self-energy [which can be considered as the energy of the Kelvin waves $\left.\mathcal{E}_{\mathrm{KW}}(k)\right]$ and inter-vortex energies [which should be associated with the hydrodynamic energy $\left.\mathcal{E}_{\mathrm{HD}}(k)\right]$ are balanced. This corresponds to condition in the $\mathbf{k}$-space,

$$
\mathcal{E}_{\mathrm{HD}}(k) \simeq \mathcal{E}_{\mathrm{KW}}(k),
$$

in the range (9). For $k>k_{\mathrm{amp}}$, Kelvin waves can propagate on an individual vortex line without approaching to (and being influenced by) the adjacent vortex lines. In the other words, the range $k>k_{\text {amp }}$ is dominated by the Kelvin wave turbulence and the role of the eddy component will be clarified below.

As we see, our corrected scenario which takes into account that the eddy/wave crossover occurs over a finite range of scales predicts a bottleneck value which is in between of the values obtained by assuming sharp transitions at the scales $\ell$ and $\ell \sqrt{\Lambda}$, respectively. 


\section{FINITE CROSSOVER RANGE MODEL}

The goal of this section is to relax the simplified assumption that the bottleneck happens at some sharp crossover scale and to describe in a simple manner the transition regimes around the characteristic scales, introduced in the previous Section. The first step in this direction is to revise the differential approximation for the cascades of turbulent energy; this is done in the following Subsection.

\section{A. Differential approximation for the cascades of turbulent energy}

The energy spectrum $\mathcal{E}(k, t)$ of isotropic turbulence can be described by the continuity equation

$$
\frac{\partial \mathcal{E}(k, t)}{\partial t}+\frac{\partial \varepsilon(k, t)}{\partial k}=0
$$

where $\varepsilon(k, t)$ is the turbulent energy flux over scales. In the stationary case this equation simplifies to the requirement of the constancy of the energy flux in the so-called inertial interval, where both energy pumping and energy dissipation can be neglected:

$$
\varepsilon(k)=\varepsilon .
$$

In order to describe a stationary spectrum $\mathcal{E}(k)$ one needs to know how $\varepsilon(k)$ depends on $\mathcal{E}(k)$. For simplicity in this paper we will use reasonably simple differential models, that describe the turbulent energy cascades of hydrodynamic (HD) and Kelvin wave (KW) turbulence at least qualitatively and sometimes even semi-quantitative.

The first differential equation model for HD was first proposed by Leith in 1967 [21] and was recently studied in [22]:

$$
\varepsilon_{\mathrm{HD}}(k)=-\frac{1}{8} \sqrt{k^{11} \mathcal{E}_{\mathrm{HD}}(k)} \frac{d}{d k} \frac{\mathcal{E}_{\mathrm{HD}}(k)}{k^{2}} .
$$

Here $\varepsilon_{\mathrm{HD}}(k)$ is the energy flux carried by HD turbulence. For Eq. (13b), the factor $\frac{1}{8}$ reproduces a numerical coefficient that reasonably fits the experimentally observed value of the Kolmogorov constant.

Generic HD spectrum with a constant energy flux was found in [22] as a solution to the equation $\varepsilon_{\mathrm{HD}}(k)=\varepsilon=$ const:

$$
\mathcal{E}_{\mathrm{HD}}(k)=k^{2}\left[\frac{24 \varepsilon}{11 k^{11 / 2}}+\left(\frac{T}{\pi \rho}\right)^{3 / 2}\right]^{2 / 3} .
$$

The large $k$ range describes a thermalized part of the spectrum with equipartition of energy characterized by an effective temperature $T$, namely, $T / 2$ of energy per a degree of freedom, thus, $\mathcal{E}_{k}=T k^{2} / \pi \rho$. At low $k$, Eq. (13a) coincides with the K41 spectrum:

$$
\mathcal{E}_{\mathrm{HD}}(k)=(24 / 11)^{2 / 3} \varepsilon^{2 / 3} k^{-5 / 3} .
$$

For Kelvin turbulence, the differential approximation model was suggested in [24]. In a way similar to Eq. (12), we suggest here a differential approximation for the energy flux, carried by the Kelvin waves:

$$
\varepsilon_{\mathrm{KW}}(k)=-\frac{5}{7} \frac{(k \ell)^{8} \mathcal{E}_{\mathrm{KW}}^{4}(k)}{\Lambda^{5} \kappa^{7}} \frac{d \mathcal{E}_{\mathrm{KW}}(k)}{d k} .
$$

Note that this form is slightly less general than the one of [24] because it does not take into account conservation of the waveaction. However, it is simpler which allows a more detailed analytical treatment.

In the stationary case, equation $\varepsilon_{\mathrm{KW}}(k)=\varepsilon=$ const has the solution

$$
\mathcal{E}_{\mathrm{KW}}(k)=\left[\frac{\Lambda^{5} \kappa^{7}}{\ell^{8}} \frac{\varepsilon}{k^{7}}+\left(\frac{T}{\pi \rho}\right)^{5}\right]^{1 / 5} .
$$

This solution changes from KW-spectrum for small $k$ :

$$
\mathcal{E}_{\mathrm{KW}}(k) \simeq \Lambda\left(\kappa^{7} \varepsilon / \ell^{8}\right)^{1 / 5} k^{-7 / 5},
$$

to the thermodynamically equilibrium solution with equipartition of energy (Rayleigh-Jeans spectrum)

$$
\mathcal{E}_{\mathrm{KW}}(k)=T / \pi \rho,
$$

for large $k$. The factor $-\frac{5}{7}$ in Eq. (14) is chosen such to reproduce in Eq. (15b) the numerical coefficient equal to unity. The actual value of this factor is still not established with a reasonable accuracy, see, e.g. [23].

When the eddy (HD) and KW turbulence coexist, both models should work together in such a manner that for small $k$ the HD spectrum should be recovered, while for large $k$ the $\mathrm{KW}$ spectrum should be the only one:

$$
\mathcal{E}(k)= \begin{cases}\mathcal{E}_{\mathrm{HD}}(k), & k \ll 1 / \ell, \\ \mathcal{E}_{\mathrm{KW}}(k), & k \gg 1 / \ell .\end{cases}
$$

A way to reach this physical requirement is presented in the following section.

\section{B. A unified model for the total eddy-wave energy flux}

A relatively simple model of turbulence with two types of motions, random eddies and Kelvin waves, is as follows. The two types of motion coexist and interact in the extended crossover range in the following sense:

a. The total turbulent energy density $\mathcal{E}(k)$ and the total energy flux over scales, $\varepsilon(k)$, consist of two respective parts:

$$
\begin{aligned}
\mathcal{E}(k) & =\mathcal{E}_{\mathrm{HD}}(k)+\mathcal{E}_{\mathrm{KW}}(k), \\
\varepsilon(k) & =\widetilde{\varepsilon}_{\mathrm{HD}}(k)+\widetilde{\varepsilon}_{\mathrm{KW}}(k) ;
\end{aligned}
$$

where energy fluxes $\widetilde{\varepsilon}_{\mathrm{HD}}(k)=\varepsilon_{\mathrm{HD}}(k)+\varepsilon_{\mathrm{HD}}^{\mathrm{KW}}$ and $\widetilde{\varepsilon}_{\mathrm{KW}}(k)=$ $\varepsilon_{\mathrm{KW}}(k)+\varepsilon_{\mathrm{KD}}(k)$ have additional contributions $\varepsilon_{\mathrm{HD}}(k)$ and $\varepsilon_{\mathrm{KW}}^{\mathrm{HD}}(k)$ that originate from influence of $\mathrm{KW}$ on the HD-energy flux and vise versa. 
b. Continuity equations (11a) for the energy densities have to be supplemented by additional terms $\pm F(k)$, that describe energy exchange between two types of motion:

$$
\begin{gathered}
\frac{\partial \mathcal{E}_{\mathrm{HD}}(k, t)}{\partial t}+\frac{\partial \widetilde{\varepsilon}_{\mathrm{HD}}(k, t)}{\partial k}=-F(k, t), \\
\frac{\partial \mathcal{E}_{\mathrm{KW}}(k, t)}{\partial t}+\frac{\partial \widetilde{\varepsilon}_{\mathrm{KW}}(k, t)}{\partial k}=F(k, t),
\end{gathered}
$$

c. Cross-contributions to the energy fluxes, $\varepsilon_{\mathrm{HD}}^{\mathrm{KW}}(k)$ and $\varepsilon_{\mathrm{KW}}^{\mathrm{HD}}(k)$ are modeled in the linear approximation with respect of the influential energies (i.e. the HD energy influencing the KW flux and vice versa) :

$$
\begin{aligned}
\varepsilon_{\mathrm{HD}}^{\mathrm{KW}} & (k)=\mathcal{D}_{\mathrm{HD}}\left\{\mathcal{E}_{\mathrm{HD}}\right\} d\left[\mathcal{E}_{\mathrm{KW}}(k) / k_{*}^{2}\right] / d k^{2}, \\
\varepsilon_{\mathrm{KW}}^{\mathrm{KW}}(k) & =\mathcal{D}_{\mathrm{KW}}\left\{\mathcal{E}_{\mathrm{KW}}\right\} d\left[\mathcal{E}_{\mathrm{HD}}(k) / k^{2}\right] / d k^{2},
\end{aligned}
$$

with some wave-vector $k_{*}$ which will be clarified later. Differential form of these contributions follows from physical hypothesis that these terms should disappear (or became much smaller and can be neglected) when the influential subsystem is in thermodynamical equilibrium, i.e. when $\mathcal{E}_{\mathrm{HD}} \propto k^{2}$ and $\mathcal{E}_{\mathrm{KW}} \propto k^{0}=$ const. Functionals of the corresponding energies, $\mathcal{D} \ldots\{\ldots\}$, will be modeled by dimensional reasoning exactly in the way, how equations (12) and (14) for the fluxes have been formulated. Resulting equations for $A$ can be written in the form:

$$
\begin{aligned}
\mathcal{D}_{\mathrm{HD}}\left\{\mathcal{E}_{\mathrm{HD}}\right\} & =C_{\mathrm{HD}} \sqrt{k^{11} \mathcal{E}_{\mathrm{HD}}(k)}, \\
\mathcal{D}_{\mathrm{KW}}\left\{\mathcal{E}_{\mathrm{KW}}\right\} & =C_{\mathrm{KW}}(k \ell) k_{*}^{2} \mathcal{E}_{\mathrm{KW}}^{4}(k) \kappa^{-7},
\end{aligned}
$$

where $C_{\mathrm{HD}}$ is a dimensionless parameter and $C_{\mathrm{KW}}(k \ell)$ is a dimensionless function of $k \ell$, that will be chosen below in Eq. (23).

d. The energy distribution between the counterpart components depends only on $k$ and for simplicity is assumed to be independent of the level of turbulence excitations:

$$
\begin{aligned}
\mathcal{E}_{\mathrm{HD}}(k, t) & =g(k \ell) \mathcal{E}(k, t), \\
\mathcal{E}_{\mathrm{KW}}(k, t) & =[1-g(k \ell)] \mathcal{E}(k, t),
\end{aligned}
$$

were we introduced (only) $k \ell$-dependent blending function $g(k \ell)$ which will be explained below.

e. Resulting model for the total energy flux $\varepsilon(k)$. Adding the two Eqs. (18) and using Eqs. (17), one yields the continuity Eq. (11a) in which $\varepsilon(k)$ is given by Eq. (17b). In such a way the unknown function $F(k, t)$ disappears from the game. Together with Eqs. (12), (14), (17b), (19), (20) and (21) this finally gives:

$$
\begin{aligned}
\varepsilon(k)=-\{ & \frac{1}{8} \sqrt{k^{11} g(k \ell) \mathcal{E}(k)} \\
+ & \left.\frac{5}{7} \frac{(k \ell)^{8} k_{*}^{2}[1-g(k \ell)]^{4} \mathcal{E}(k)^{4}}{\Lambda^{5} \kappa^{7}}\right\} \times \\
& \frac{d}{d k}\left\{\mathcal{E}(k)\left[\frac{g(k \ell)}{k^{2}}+\frac{1-g(k \ell)}{k_{*}^{2}}\right]\right\} .
\end{aligned}
$$

In the derivation of this equation we took

$$
C_{\mathrm{HD}}=-1 / 8, \quad C_{\mathrm{KW}}(k \ell)=-5(k \ell)^{8} / 7 \Lambda^{5} .
$$

Only with this choice the resulting Eq. (22) for $\varepsilon(k)$ is proportional to $d\left[\mathcal{E}(k) / k^{2}\right] / d k$ and $\varepsilon(k)$ vanishes in the thermodynamical equilibrium with $\mathcal{E}(k) \propto k^{2}$, as one should expect.

Equation (23) contains yet unknown blending function $g(k \ell)$ which will be discussed in the next section.

\section{Separation of the eddy and wave motions}

In order to find a qualitative form of the blending function we consider a system of locally (in the vicinity of some point $\boldsymbol{r}_{0}$ ) near-parallel vortex lines, separated by mean distance $\ell$ and supply them by index $j$. Notice that in principle the same vortex line can go far away and come close to $\boldsymbol{r}_{0}$ several times. To avoid this problem one should assign the same vortex line a different index $j$ if it leaves (or enters) the ball of radius $\ell \sqrt{\Lambda}$ centered at $\boldsymbol{r}_{0}$. Each vortex line (with zero radius $a$ ) produces a velocity field $\boldsymbol{v}_{j}(\boldsymbol{r})$, which can be found by the Biot-Savart Law (A1).

The total kinetic energy $E=\frac{1}{2} \sum_{i, j}\left\langle\boldsymbol{v}_{i} \cdot \boldsymbol{v}_{j}\right\rangle$ can be divided into two parts, $E=E_{1}+E_{2}$, where

$$
E_{1} \equiv \frac{1}{2} \sum_{j}\left\langle v_{j}^{2}\right\rangle, \quad E_{2} \equiv \frac{1}{2} \sum_{i \neq j}\left\langle\boldsymbol{v}_{i} \cdot \boldsymbol{v}_{j}\right\rangle=\sum_{i<j}\left\langle\boldsymbol{v}_{i} \cdot \boldsymbol{v}_{j}\right\rangle .
$$

The same subdivision can be made also for the energy density in the (one-dimensional) $k$-space, $\mathcal{E}(k)=\mathcal{E}_{1}(k)+$ $\mathcal{E}_{2}(k)$, with two terms, that can be found via $\boldsymbol{k}$-Fourier components of the velocity fields $\boldsymbol{v}_{j}(\boldsymbol{k})$ in the way, similar to Eq. (24). Now our idea is as follows: energy $\mathcal{E}_{1}(k)$ is defined by the form of the individual vortex lines, that is determined by the Kelvin waves, while energy $\mathcal{E}_{2}(k)$ depends on correlations in the form of different vortices, that produce collective, hydrodynamic type of motions. Therefore $\mathcal{E}_{1}(k)$ can be associated with the Kelvin wave energy, $\mathcal{E}_{1}(k) \Rightarrow \mathcal{E}_{\mathrm{KW}}(k)$, while $\mathcal{E}_{1}(k)$ has to be associated with the hydrodynamic energy, $\mathcal{E}_{2}(k) \Rightarrow \mathcal{E}_{\mathrm{HD}}(k)$. This allows one to conclude that

$$
g(k \ell)=\left[1+\mathcal{E}_{1}(k) / \mathcal{E}_{2}(k)\right]^{-1} .
$$

The rest is technicalities presented in Appendix A, where we concluded that in practical calculations it is reasonable to use analytical form $g(k \ell)$ of the blending function

$$
g(k \ell)=g_{0}[0.32 \ln (\Lambda+7.5) k \ell]
$$

where

$$
g_{0}(k \ell)=\left[1+\frac{(k \ell)^{2} \exp (k \ell)}{4 \pi(1+k \ell)}\right]^{-1}
$$




\begin{tabular}{||c||c|c|c|c|c||}
\hline \hline- & 1 & 2 & 3 & 4 & 5 \\
\hline$\Lambda$ & $\begin{array}{c}k_{\text {vel }} \ell \\
\text { Eq. (1b) }\end{array}$ & $\begin{array}{c}k_{\text {en }} \ell \\
\text { Eq. (27) }\end{array}$ & $\begin{array}{c}k_{\text {amp }} \ell \\
\text { Eq. (7c) }\end{array}$ & $\begin{array}{c}k_{\mathrm{f}} \ell \\
\text { Numerics }\end{array}$ & $\begin{array}{c}\epsilon \times 10^{-3} \\
\text { self-cons. }\end{array}$ \\
\hline \hline 10 & 0.45 & 2.3 & 0.76 & 24.4 & 5.2 \\
\hline 30 & 0.26 & 1.8 & 0.64 & 23.6 & 2.1 \\
\hline $10^{2}$ & 0.14 & 1.4 & 0.52 & 22.8 & 0.30 \\
\hline $10^{3}$ & 0.045 & 0.96 & 0.35 & 22.5 & $1.2 \times 10^{-3}$ \\
\hline \hline
\end{tabular}

TABLE I: Comparison of the energy-, velocity-, amplitudeand flux-crossover scales for typical experimental values $\Lambda=$ 10 and 30 and unrealistically large values $\Lambda=100$ and $10^{3}$. Values of $k_{\mathrm{f}}$ depend on $\epsilon$ defined in a self-consistent way, explained in Sec. III D 3

\section{Comparison of various crossover scales}

With the proposed blending function we can introduce another cross-over scale $k_{\text {en }} \ell$ by comparing HD and KW energies, at $k_{\mathrm{en}} \ell$ they are equal: $g\left(k_{\mathrm{en}} \ell\right)=1 / 2$. As follows from Eqs. (26), $k_{\text {en }} \ell$ has very weak, logarithmical dependence on $\Lambda$ :

$$
k_{\mathrm{en}} \ell \simeq 6.64 / \ln (\Lambda+7.5),
$$

presented in the second column in Table. I.

First column of this table displays velocity-crossover scales, given by Eq. $(1 \mathrm{~b}): k_{\mathrm{vel}} \ell \simeq \sqrt{2 / \Lambda}$, while in the column 3 one finds the amplitude crossover $k_{\text {amp }} \ell$ Eq. $(7 \mathrm{c})$, scale at which the formally computed with the KW spectrum amplitude of Kelvin waves reaches intervortex distance. The fourth column of Table. I displays fluxcrossover scale, $k_{\mathrm{ff}} \ell$ at which the contribution of the eddyand Kelvin-wave turbulence to the energy flux in the $k$ space become equal. This scale is introduced below in Sec. III D.

Important message is that although theoretically, in the limit $\Lambda \rightarrow \infty$ (see Appendix), $k_{\text {en }} \ll k_{\text {amp }}$, in the region $10 \lesssim \Lambda \lesssim 30$ these scales are close, moreover, due to numerical prefactors $k_{\mathrm{en}}>k_{\mathrm{amp}}$. Therefore the fractal structure of the vortex lines, described in Sec. II C, does not appear which allows us to use in the actual region $10 \lesssim \Lambda \lesssim 30$ the proposed simple blending function (26b).

\section{Turbulent energy spectra in the differential approximation}

\section{Dimensionless representation}

At the end of the day we are left with solving the ODE (22), where the blending function is $g(k \ell)$, Eqs. (26b). At this stage of research it is reasonable to nondimensionalize physical quantities introducing

$$
x=k \ell, \quad e(x)=\frac{\ell}{\kappa^{2}} \mathcal{E}(x), \quad \epsilon=\frac{\ell^{4}}{\kappa^{3}} \varepsilon .
$$

In particular, with this normalization the onedimensional energy spectra HD and KW, Eqs. (13) and (15b), take the form

$$
\begin{aligned}
e_{\mathrm{HD}}(x) & =(24 / 11)^{2 / 3} \epsilon^{2 / 3} x^{-5 / 3}, \\
e_{\mathrm{HD}}(x, T) & =x^{2}\left[\frac{24}{11} \frac{\epsilon}{x^{11 / 2}}+T^{3 / 2}\right]^{2 / 3}, \\
e_{\mathrm{KW}}(x) & =\Lambda \epsilon^{1 / 5} x^{-7 / 5},
\end{aligned}
$$

where $T$ is non-dimensional temperature. And the ODE (22) to solve [with the boundary condition $e(x) \rightarrow$ $e_{\mathrm{KW}}(x)$ for $x \gg 1$ ] becomes

$$
\begin{aligned}
& \epsilon=-\{ \frac{1}{8} \sqrt{x^{11} g(x) e(x)} \\
&+\left.\frac{5}{7} \frac{x^{8} x_{\mathrm{en}}^{2}}{\Lambda^{5}}[1-g(x)]^{4} e^{4}(x)\right\} \times \\
& \frac{d}{d x} e(x)\left[\frac{g(x)}{x^{2}}+\frac{1-g(x)}{x_{\mathrm{en}}^{2}}\right] .
\end{aligned}
$$

Here we made the natural choice that the crossover scale $k_{*}$ between two types of thermodynamic equilibrium is $k_{\mathrm{en}}$, the scale where energies of two types of motion are the same.

\section{2. $\Lambda$-dependence of the energy spectra}

An instructive solution $e(x)$ with $\Lambda=30$ and $\epsilon=$ $2.110^{-3}$ is shown in Fig. 1, left, as a (black) solid line. One sees that this solution for $x \lesssim x_{\mathrm{en}} \simeq 1.83$ follows the thermolized HD spectrum $e_{\mathrm{HD}}(x, T)$ [given by Eq. (29b) with properly chosen $T$ ] shown as a dotted (cyan) line. An important observation is that the pseudo-thermolized part of the spectrum is very pronounced in the region $x \gtrsim 0.5$ where it is very different from the K41 spectra of HD turbulence $e_{\mathrm{HD}}(x) \propto x^{-5 / 3}$ shown as a dashed (blue) line. For $x \gtrsim x_{\mathrm{fl}}$ the solution practically coincides with the pure $\mathrm{KW}$ spectrum $e_{\mathrm{KW}}(x),(29 \mathrm{c})$ shown as a dashed (green) line. Important, that the crossover scale $x_{\mathrm{ff}} \simeq 23.6$, at which the total energy flux consists of $50 \%$ of HD- and $50 \%$ of $\mathrm{KW}$-fluxes, is much larger that $x_{\text {en }} \simeq 1.83$, at which a half of the total energy is carried by HD and half by KW motions. To make this evident we plotted in Fig. 1, right, the partial HD- and KWenergy fluxes vs. $x$. They become equal at $x_{\mathrm{fl}}$, which for $\Lambda=100$ and $\epsilon=310^{-4}$ is around 23.6.

In the intermediate region $x_{\mathrm{en}}<x<x_{\mathrm{fl}}$ the energy consists mostly of the KW energy, while the energy flux is carried mostly by the HD motions. Explanation to this observation is simple: as follows from Eq. (30) the $\mathrm{HD}$ motions are more effective (in factor $\sim \Lambda^{5} / x^{9 / 2}$ ) in support of the energy flux then the KW turbulence. Because the main part of the energy flux is taken by the $\mathrm{HD}$ motions, the KW energy spectrum (and therefore the total one) is close to the flux-less $\mathrm{KW}$-solution: thermodynamic equilibrium (15c), $\mathcal{E}_{\mathrm{KW}}=$ const. For $x>x_{\mathrm{fl}}$, 

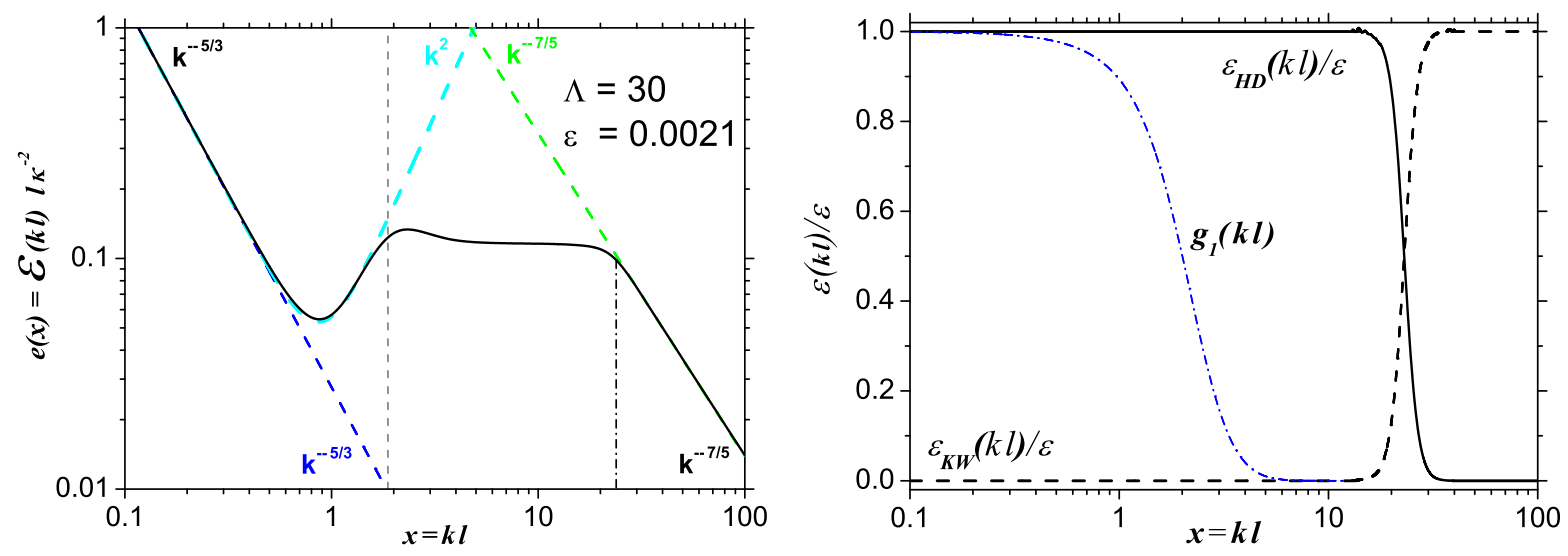

FIG. 1: Left panel: Solid (black) line represents typical total dimensionless energy spectrum $e(x)$ obtained by numerical solution of the ODE (30) with $\Lambda=30$ and self-consistent value of $\epsilon=0.0021$, found in Sec. IIID 3 . Dashed (blue) line corresponds to the K41 energy spectrum $e_{\mathrm{HD}}(x) \propto x^{-5 / 3}$ with constant energy flux, dashed (cyan) line is general HD spectrum $e_{\mathrm{HD}}(x, T=0.042)$, dashed (green) line is the energy spectrum of Kelvin waves $e_{\mathrm{KW}}(x) \propto x^{-7 / 5}$. Vertical dashed (gray) line shows $x_{\mathrm{en}}$. Vertical dot-dashed (brown) line shows position $x_{\mathrm{fl}}$, where $\epsilon_{\mathrm{HD}}\left(x_{\mathrm{fl}}\right)=\epsilon_{\mathrm{KW}}\left(x_{\mathrm{fl}}\right)$. Right panel: Partial energy fluxes $\epsilon_{\mathrm{HD}}(x) / \epsilon$ (solid line) and $\epsilon_{\mathrm{KW}}(x) / \epsilon$ (dashed line) obtained by numerical solution of the ODE (30) with $\Lambda=30$ and $\epsilon=0.0021$. Dot-dashed (blue) line represents $g(x)$.
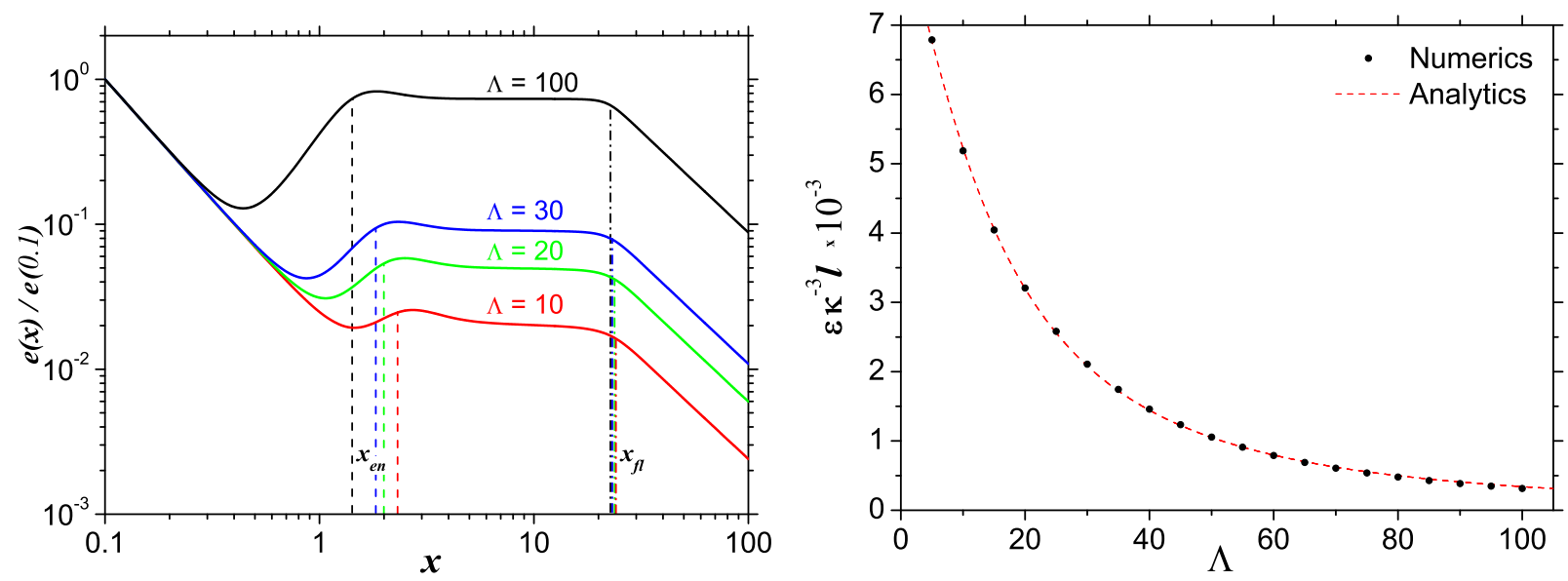

FIG. 2: Normalized non-dimensional energies $e(x)$ at $\Lambda=10,20,30$ and 100 (left) and $\Lambda$-dependence of the self-consistent energy flux $\epsilon$. Vertical dashed (gray) line shows $x_{\mathrm{en}}$. Vertical dash-dotted lines indicate positions of $x_{\mathrm{fl}}$ (in red, green and blue for corresponding $\epsilon$ ).

both the energy and the energy flux are carried by the KW motions. Therefore the total energy spectrum coincides with the KW cascade solution. For larger values of $\epsilon$ the flux-crossover scale goes to the smaller values of $k$, see Fig. 2, remaining nevertheless larger than $k_{\mathrm{en}}$.

For smaller value of $\Lambda$ qualitative behavior of $\mathcal{E}(x)$ remains the same, just different parts of the spectra (with larger values of self-consistent values of $\epsilon$ ) become less pronounced.

\section{Self-consistent estimate of the dimensionless energy flux}

Energy spectra, Figs. 1 and 2, which we obtained in the proposed differential approximation are quite similar to those suggested under the assumption of sharp crossover, see Fig. 1 in Ref. [19]: for small $k$ they coincide with the HD spectrum, including the bottleneck part with (almost) thermalized part $\mathcal{E} \propto k^{2}$, while for large $k$ the spectrum follows the KW spectrum $\propto k^{-7 / 5}$. The only difference is that with sharp crossover the thermalized part of the HD-spectrum is matched with $\mathrm{KW}$-spectrum at some $k=k_{*}$, while in the differential approximation with a smooth blending function there is essential intermediate region (about one decade) $k_{\text {en }}<k<k_{\mathrm{fl}}$, with (almost) KW-thermalized part $\mathcal{E}=$ const. This leads to an essential difference in the estimates of the vorticity $\left\langle\omega^{2}\right\rangle$ and as a result in the estimates of the effective viscosity $\nu^{\prime}$, a parameter that can be measured (implicitly) in experiments. Indeed, in the models with the sharp 
crossover one estimates $\left\langle\omega^{2}\right\rangle$ in Eq. (3) as $k_{*}^{3} \mathcal{E}_{\mathrm{HD}}\left(k_{*}\right)$ and then equates $\mathcal{E}_{\mathrm{HD}} \simeq \mathcal{E}_{\mathrm{KW}}$, because in this model the HDenergy "transforms" into the KW-energy at the position of the sharp crossover.

In the "continuous" model presented above one has to account for a wide region between $k_{\mathrm{en}}$ and $k_{\mathrm{ff}}$, where the flux is supported by the HD turbulence, while the energy is dominated by the Kelvin waves. Therefore $\left\langle\omega^{2}\right\rangle$ can be estimated similarly to Eq. (3) as $2 \int k^{2} \mathcal{E}_{\mathrm{HD}}(k) d k \simeq$ $k_{\mathrm{en}}^{3} \mathcal{E}_{\mathrm{HD}}\left(k_{\mathrm{en}}\right)$, but now $\mathcal{E}_{\mathrm{HD}}\left(k_{\mathrm{en}}\right)$ cannot be estimated based on the $-7 / 5$ Kozik-Svistunov spectrum because, as one sees in Fig. 2, $\mathcal{E}_{\mathrm{HD}}$ is much lower at this point. As a result, at the same energy flux the rms. vorticity $\sqrt{\left\langle|\omega|^{2}\right\rangle}$ occurs to be essentially smaller then that in the sharpcrossover models and the effective viscosity is larger. In our approach the rms vorticity can be found more accurately by numerical calculation of the integral in Eq. (3) with spectrum $\mathcal{E}_{\mathrm{HD}}(k) \simeq g(k \ell) \mathcal{E}(k)$, where the blending function is given by Eqs. (26):

$$
\left\langle|\omega|^{2}\right\rangle=2 \int_{k_{\min }}^{\infty} k^{2} g(k \ell) \mathcal{E}(k) d k,
$$

where $k_{\min }$ is the lower cutoff of the inertial interval. Using relation $\left\langle|\omega|^{2}\right\rangle=\kappa^{2} / \ell^{4}$ and normalization (28) one finds from Eq. (31a) in the limit $k_{\min } \rightarrow 0$ :

$$
1=2 \int_{0}^{\infty} x^{2} g(x) e(x) d x .
$$

Due to $\epsilon$ dependence of the energy spectrum $e$, this relation gives self-consistent a estimate of the dimensionless energy flux $\epsilon$, which is according to Eqs. (5) and (28) is nothing else, but $\nu^{\prime} / \kappa$. Resulting dependence $\epsilon$ vs. $\Lambda$ is shown in Fig. 2, right, as solid line. For convenience we approximate this dependence (in the actual interval $\Lambda<100)$ analytically:

$$
\epsilon=\frac{\nu^{\prime}}{\kappa}=\frac{8.65}{10^{3}+45.8 \Lambda+1.98 \Lambda^{2}},
$$

shown in Fig. 2, right, as dashed line. Equation (32) reproduces numerical dependence $\epsilon(\Lambda)$ with accuracy better than $1.5 \%$ for $\Lambda<50$ and better than $8 \%$ for $50<\Lambda<100$.

Notice, that found values of $\epsilon=\nu^{\prime} / \kappa$ for $\Lambda=30$ is 0.021 which is quite close to the experimentally reported value $\nu^{\prime} \simeq 0.003 \kappa$ in ${ }^{4} \mathrm{He}$ experiments at low temperatures. A relationship between out model and experiments will be discussed below.

\section{E. Decay of quantum turbulence with the bottleneck energy accumulation}

Having in mind experiments with decaying superfluid turbulence, like the ones in [25], it is important to discuss how the bottleneck energy accumulation influences the decay of energy and vorticity in time. For this, we divide the total HD energy

$$
E_{\mathrm{HD}}=\int_{k_{\min }}^{\infty} d k \mathcal{E}_{\mathrm{HD}}(k)
$$

into a sum of two parts:

$$
E_{\mathrm{HD}}=E_{\mathrm{HD}}^{\mathrm{K} 41}+E_{\mathrm{HD}}^{\mathrm{TE}},
$$

the energy $E_{\mathrm{HD}}^{\mathrm{K} 41}$ associated with the K41 part of energy spectra $\mathcal{E}_{\mathrm{HD}} \propto k^{-5 / 3}$, and the energy $E_{\mathrm{HD}}^{\mathrm{TE}}$ associated with the thermodynamic equilibrium (TE) part of the spectrum $\mathcal{E}_{\text {HD }} \propto k^{2}$. For our model:

$$
\begin{aligned}
E_{\mathrm{HD}}^{\mathrm{K} 41} & =\int_{k_{\min }}^{k_{\mathrm{TE}}} d k \mathcal{E}_{\mathrm{HD}}(k), \\
E_{\mathrm{HD}}^{\mathrm{TE}} & =\int_{k_{\mathrm{TE}}}^{\infty} d k \mathcal{E}_{\mathrm{HD}}(k),
\end{aligned}
$$

where $k_{\mathrm{TE}}$ is the crossover scale between K41 and TE parts of the energy spectra corresponding to the position where $\mathcal{E}_{\mathrm{HD}}(k)$ is minimal. For $\Lambda=30, k_{\mathrm{TE}} \approx 1 / \ell$, see Fig. 1, left. The K41-energy, $E_{\mathrm{HD}}^{\mathrm{K} 41}$, is dominated by the outer region of the $k$-space, $k \gtrsim k_{\min }$, while the TEenergy is determined by effectively the largest $k \simeq k_{\text {en }}$ of the HD motions (Fig. 1, left). In the experiment [25], $k_{\text {min }}$ is below $1 \mathrm{~cm}^{-1}$, which is less than $k_{\text {en }} \simeq 2 / \ell$ by one or two orders of magnitude. Then, the experiment [25] allows to estimate the ratio $E_{\mathrm{HD}}^{\mathrm{TE}} / E_{\mathrm{HD}}^{\mathrm{K} 41}$ in the proposed framework, and it varies from a few percents for small times to about $15-20 \%$ at the latest times of the decay measurements. For us this means that with an acceptable accuracy one can neglect the contribution of $E_{\mathrm{HD}}^{\mathrm{TE}}$ in Eq. (33b).

Moreover, even when kept in Eq. (33b), the energy $E_{\mathrm{HD}}^{\mathrm{TE}}$ does not appreciably affect on the decay rate of $E_{\mathrm{HD}}^{\mathrm{K} 41}$ energy due to a large scale separation $\left(k_{\min } \ll k_{\text {en }}\right)$. The decay rate of $E_{\mathrm{HD}}^{\mathrm{K} 41}$ is determined by the energy flux $\varepsilon=$ $-d E_{\mathrm{HD}}^{\mathrm{K} 41} / d t$ at the scale of the energy pumping, i.e. at the outer scale $k=k_{\min }$. The flux itself is proportional to $\mathcal{E}_{\mathrm{HD}}(k)^{3 / 2} \sim\left(E_{\mathrm{HD}}^{\mathrm{K} 41} / k_{\mathrm{min}}\right)^{3 / 2}$. For systems with the time independent $k_{\min }$, as it is in [25], this gives the well known result for the late-time free-decaying HD turbulence:

$$
E_{\mathrm{HD}}^{\mathrm{K} 41}(t) \propto t^{-2},
$$

and the time-evolution of the energy flux

$$
\varepsilon(t) \propto t^{-3} .
$$

According to Eq. (28), $\varepsilon(t)=\epsilon \kappa^{3} / \ell^{4}(t)$ with the timeindependent self-consistent dimensionless energy flux $\epsilon$, which depends only on $\Lambda$. This gives $\ell(t) \propto t^{3 / 4}$. Therefore, the vortex line density must decay in the standard manner:

$$
L=1 / \ell^{2} \propto t^{-3 / 2},
$$


in spite of the accumulation of energy $E_{\mathrm{HD}}^{\mathrm{TE}}$ near the crossover scale $k_{\text {en }}$.

Notice, that energy $E_{\mathrm{HD}}^{\mathrm{TE}}(t)$ decays slower than $E_{\mathrm{HD}}^{\mathrm{K} 41}(t) \propto t^{-2}$. Indeed, in our model the dimensionless energy $E_{\mathrm{HD}}^{\mathrm{TE}} \ell^{2} / \kappa^{2}$ (cf. Eq. (28)) is dominated by the time independent scale $x_{\text {en }}$ and, hence, by itself is time independent. Therefore,

$$
E_{\mathrm{HD}}^{\mathrm{TE}}(t) \propto \ell^{-2} \propto t^{-3 / 2} .
$$

One concludes that $E_{\mathrm{HD}}^{\mathrm{TE}}$ energy is "decoupled" from the decay process of $E_{\mathrm{HD}}^{\mathrm{K} 41 \mathrm{D}}$ energy and does not affect the decay law (34c) of the vortex line density until to the very late stage of the decay, when the intervortex distance approaches the outer scale of turbulence and the entire model fails.

\section{Summary and Discussion}

In this paper, we revised the theory of the bottleneck crossover from the classical K41 cascade to the Kelvin wave cascade. In its previous form, transition from the eddy to the wave cascades was assumed to occur sharply at the scale $\ell$. The simple fact that the wave interactions are less efficient for the turbulent cascade than the hydrodynamic eddies immediately yields prediction for the bottleneck accumulation near the crossover scale. However, the bottleneck strength is rather sensitive to the details of the crossover region. In the present paper, we take into account that there exists a finite range where eddies and waves coexist and affect each other, making the crossover more gradual. As a result, the bottleneck in such a case is milder than in the model with the sharp crossover. To model the gradual transition range, we have employed a simplified turbulence model which is based on the differential approximation models of Leith type for the HD and KW components. Importantly, this model allows to make predictions for the realistic experimental values $\Lambda$ in the range from 10 to 30 , rather than making asymptotical predictions for the case $\Lambda \rightarrow \infty$. This appears to be important because, e.g., the asymptotic theory gives $k_{\text {amp }} \gg k_{\text {en }}$ whereas for $\Lambda$ in the range from 10 to 30 we have $k_{\mathrm{amp}} \lesssim k_{\mathrm{en}}$. As a result, for the experimentally important situations there is no range with equipartition of the eddy and the wave energies given by (10). For similar reasons, the theory of crossover [20] which fits three asymptotic ranges into a single decade of scales is rather unrealistic (leaving aside the issue about the role of reconnections which we mentioned before).

One may experience some problems trying to imagine any HD components at $k \ell>2 \pi$, where the wavelength becomes larger than the intervortex distance, and even come to an idea that $g(k \ell)$ must become zero sharply at $k \ell=2 \pi$, or generally, $k \ell \sim 1$. Our model is based on a (reasonable) hypothesis that the HD motions are identified with the coherent part of different vortex line motions. In such an approach there is no formal limitation for the value of $k$ from above. At $k \ell>1$ the velocity produced by $k$-distortion of a given vortex line in the position of another $\ell$-separated line, which is the reason for correlations in their motions, decays exponentially with $k \ell$. That is why our blending function, which measures the fraction of the HD motions, decays exponentially with $k \ell$. The actual hypothesis in this place is that even when $g(k \ell) \ll 1$ the nonlinear energy flux carried by the HD motions is governed by the same equations as for the pure HD motions when $g(k \ell)=1$. We believe this is a step forward in comparison with the oversimplified scenario of sharp crossover which, by the way, is a limiting case for our model where the blending function is just a unit-step function $g(k)=\Theta\left(k_{\mathrm{dim}}-k\right)$.

The found value of $\epsilon=\nu^{\prime} / \kappa$ for $\Lambda=30$ is 0.021 which is quite close to the experimentally reported value $\nu^{\prime} \simeq 0.003 \kappa$ in ${ }^{4} \mathrm{He}$ experiments at low temperatures. Having in mind that our model does not contain fitting parameters, optimists can consider this agreement as more than satisfactory. On the other hand, pessimists can consider any agreement with just one number as accidental. Realists should recall that the suggested model is based on a hypothesis of blending function, which was estimated without taking into the account the vectorial structure of the velocity field and, moreover, includes very important (step-like) assumption about the pairdistribution function of the vortex positions which allows one to estimate sum (A7a) as integral (A7b). Our feeling is that these approximations do not affect the results too much and a good agreement between the model and experimental values of $\nu^{\prime}$ supports the suggested model. Notice that our model predicts not only the value of $\nu^{\prime}$ but the entire energy spectrum, which consists of four parts: K41 HD energy spectrum with constant energy flux, $\mathcal{E} \propto k^{-5 / 3}$, a HD equilibrium $\mathcal{E} \propto k^{2}$, a $\mathrm{KW}$ equilibrium $\mathcal{E} \simeq$ const and a $\mathrm{KW}$-spectrum with constant energy flux, $\mathcal{E} \propto k^{-7 / 5}$. This very definite qualitative prediction calls for more detailed experimental and numerical study of the superfluid turbulence, which, as we believe, will support our model.

\section{Acknowledgements}

This work has been partially supported by the Transnational Access Programme at RISC-Linz, funded by the European Commission Framework 6 Programme for Integrated Infrastructures Initiatives under the project SCIEnce (Contract No. 026133). 


\section{APPENDIX A: ESTIMATION OF THE HD-KW BLENDING FUNCTION}

\section{Estimation of the velocity field, induced by the vortex distortion}

To estimate the HD-KW blending function $g(k \ell)$, given by Eq. (25) we consider a vortex line slightly distorted, say in $y$-direction, by a sinus with a small amplitude $A$, running along $z$-axis with the $k$-vector $k$. Then $d \boldsymbol{\ell}=$ $(0, A k \cos (k z+\phi), 1) d z$, where $\phi$ is an arbitrary phase. Each line produces velocity that can be found via BioSavart Law:

$$
\boldsymbol{v}_{j}(\boldsymbol{r})=\frac{\kappa}{4 \pi} \int_{-\infty}^{+\infty} \frac{d \boldsymbol{\ell}_{j} \times \boldsymbol{s}_{j}}{s_{j}^{3}}
$$

where $\boldsymbol{s}_{j}=\boldsymbol{r}-\boldsymbol{r}_{j}$ with $\boldsymbol{r}_{j}$ being the radius-vector pointing to the $\ell_{j}$ - the length element along the $j$-th vortex line and. The total mean density of the kinetic energy per unit mass is $E=\frac{1}{2}\left\langle|V|^{2}\right\rangle$, where $\boldsymbol{V}(\boldsymbol{r})=\sum_{j} \boldsymbol{v}_{j}(\boldsymbol{r})$ is the total velocity field, and one understands $\langle\ldots\rangle$ as the averaging with respect of the (random) vortex-line positions.

Using Eq. (A1) we can find the resulting velocity (for simplicity) in the $Z Y$-plane at distance $R$ from the line. This velocity has only one component, $v_{x}$, and it is given by:

$$
v_{x}=\frac{\kappa}{4 \pi} \int_{-\infty}^{\infty} \frac{R-A \sin (k z+\phi)+A k z \cos (k z+\phi)}{\left\{z^{2}+[R-A \sin (k z+\phi)]^{2}\right\}^{3 / 2}} d z
$$

Computing the contribution to the amplitude of the velocity variations proportional to the distortion amplitude $A$, one finds magnitude [i.e. factor in front of $\cos (k z+\phi)]$ of the velocity fluctuations:

$$
\begin{aligned}
\delta v(R)= & \frac{\kappa A}{2 \pi R^{2}} k R \times \\
& \left\{k R\left[K_{2}(k R)-K_{0}(k R)\right]-K_{1}(k R)\right\}, \Rightarrow \\
\delta v(R) \simeq & \frac{\kappa A}{2 \pi R^{2}} \sqrt{1+\frac{\pi}{2} k R} \exp (-k R),
\end{aligned}
$$

where $K_{n}$ are modified Bessel functions of the second kind. Interesting, that a simple interpolation (A3b) reproduces the exact result (A3a) with an accuracy better than $2 \%$.

Equations (A3) assume that the distance $R$ to the vortex line is large enough to be able to neglect finiteness of the core radius $a$. In the opposite limit, $R \ll a$, one can use an equation for the self-induced velocity, in which the core radius is hidden in the parameter $\Lambda=\ln (\ell / a)$ :

$$
\delta v_{\mathrm{si}}=A \Lambda k^{2} \kappa / 4 \pi
$$

Considering this equation formally as a limit for the velocity when $R \rightarrow 0$, we suggest an interpolation formula approximately valid for any $R$ :

$$
\delta v_{k}(R) \simeq \frac{\kappa A}{2 \pi} \frac{\Lambda k^{2} e^{-k R}}{2+\Lambda(k R)^{2}} \sqrt{1+\frac{\pi}{2} k R},
$$

where subscript " $k$ " reminds that this velocity is induced by vortex line, distorted by $\sin (k r)$.

\section{Velocity crossover scale}

A way to estimate the eddy-wave crossover scale is to compare the self-induced velocity filed with the velocity field produced by a different neighboring vortex line. For this goal we consider simple geometry with two sindisturbed parallel vortex lines separated by distance $\ell$ They "start to feel" each other when $\delta v \simeq \delta v$, i.e.:

$$
\delta v \simeq \delta v^{\mathrm{SI}} \Leftrightarrow \sqrt{4+2 \pi k_{\mathrm{vel}} \ell} \mathrm{e}^{-k_{\mathrm{vel}} \ell} \simeq \Lambda\left(k_{\mathrm{vel}} \ell\right)^{2} .
$$

An approximate solution to this equation (with an accuracy better than $10 \%$ for $\Lambda \geq 10$ ) is given by Eq. (1b). One can think that for the scales less than $k_{\mathrm{vel}}$, there is the cumulative effect and hydrodynamic-like behavior. For the scales larger than $k_{\text {vel }}$ the vortices does not seem to feel each other much, and the separate vortex line behavior is important, hence the Kelvin waves. Corrections to this simplest viewpoint will be discussed below.

\section{Estimate of the blending function}

Having at hand an estimate of the velocity filed (A3d) we can use Eq. (25) to find the blending function $g(k \ell)$.

a. The first step is to obtain the kinetic energy density $E_{11}(k)$ (per unit length), produced by one sindistorted (with wave-vector $k$ ) vortex line:

$$
\begin{gathered}
E_{11}(k)=\frac{\rho_{0}}{2} \iint\left\langle\left|v_{k}(R, z)\right|^{2}\right\rangle d x d y \\
\approx \rho_{0} \frac{(A \kappa \Lambda k)^{2}}{8 \pi} \int_{0}^{\infty} \frac{(1+\pi \rho / 2) e^{-2 \rho}}{\left(2+\Lambda \rho^{2}\right)^{2}} \rho d \rho \\
\approx \rho_{0} \frac{\Lambda}{8 \pi}(A \kappa k)^{2}, \text { for } \Lambda \gg 1 .
\end{gathered}
$$

Here the fluid density $\rho_{0}$ should be distinguished from the dimensionless radius $\rho=k R, R=\sqrt{x^{2}+y^{2}}$. In Eq. (A5a) we should substitute $v_{k}(R, z)=v_{k}(R) \cos (k z+$ $\phi)$ from Eq. (A3d) integrate over $(x, y)$-plane orthogonal to the mean vortex line directed along $z$, and average $\left\langle\cos ^{2}(k z+\phi)\right\rangle=\frac{1}{2}$ along $z$. Considering the integral in dimensionless polar coordinates $\rho$ and $\varphi$ after the free integration over $\varphi$, we get Eq. (A5b), in which integral can be analytically taken in the limit of large $\Lambda$ with the result (A5c). This formula can also be obtained directly from the Kelvin-wave Hamiltonian in the so-called localinduced approximation, see e.g. Eq. (5b) in [19], where one expands $\sqrt{1+|d w(z) / d z|^{2}}$ over the line distortion $w(z)=A \sin (k z)$. 
b. The second step is to find cross-kinetic energy density $E_{1 j}\left(k, \ell_{1 j}\right)$ (per unite length), proportional to the product of the velocities produced by two sin-distorted vortex lines, separated by $\ell_{1 j}$.

$$
\begin{gathered}
E_{1 j}\left(k, \ell_{1 j}\right)=\rho_{0} \int_{-\infty}^{\infty} d x \int_{-\infty}^{\infty} d y\left\langle\left|v_{k}\left(R_{1}, z\right) v_{k}\left(R_{2}, z\right)\right|\right\rangle \\
=\frac{\rho_{0}}{2}\left(\frac{\kappa A \Lambda k}{\pi}\right)^{2} \int_{0}^{\infty} \delta \widetilde{x} \int_{-\infty}^{\tilde{\ell}_{1 j} / 2} \delta \widetilde{y} F\left(\rho_{1}\right) F\left(\rho_{2}\right) e^{-\rho_{+}}, \\
F(\rho)=\frac{\sqrt{1+\pi \rho / 2}}{2+\Lambda \rho^{2}}, \quad \rho_{+}=\rho_{1}+\rho_{2} .
\end{gathered}
$$

Here $\tilde{x}=k x, \quad \widetilde{y}=k y, \quad \tilde{\ell}_{1 j}=k \ell_{1 j}, \rho_{1}=k \sqrt{x^{2}+y^{2}}$ and $\rho_{2}=k \sqrt{x^{2}+\left(y-\ell_{1 j}\right)^{2}}$. The change in the limits of integration is due to the (average) symmetry of the integrand relative to the plane $y=\ell_{1 j} / 2$. The main contribution to the integral comes from the region with $\widetilde{x} \lesssim \widetilde{\ell}_{1 j}$, which allows to simplify the integrand replacing $\rho_{2} \Rightarrow \widetilde{\ell}_{1 j}-\widetilde{y}+\widetilde{x}^{2} /\left(2 \ell_{1 j}\right)$. This clarifies the leading dependence $E_{1 j}\left(k, \ell_{1 j}\right)$ on $\ell_{1 j}$ as

$$
E_{1 j}\left(k, \ell_{1 j}\right) \propto \exp \left(-k \ell_{1 j}\right) \text {. }
$$

Algebraic improvement of this estimate cane be obtained by neglecting the $(x, y)$-dependence of $F\left(\rho_{2}\right)$ in Eq. (A6a). This procedure can be justified asymptotically for $k \ell_{1 j} \gg 1$. The resulting dependence $E_{1 j}\left(k, \ell_{1 j}\right) \propto F\left(k \ell_{1 j}\right) \exp \left(-k \ell_{1 j}\right)$ approximates the integral Eq. (A6a) with an accuracy of $10 \%$ for $k \ell_{1 j}>2$. For smaller $k \ell_{1 j}$ one has to account for $\ell_{1 j}$ dependence of the remained integral $\int_{-\infty}^{\ell_{1 j} / 2} \ldots d y \simeq(a / \Lambda) \ln \left(k \ell_{1 j}+b\right)$ with $a$ and $b$ weakly dependent on $\Lambda$. As a result we approximate (with accuracy about $25 \%$ ) the $k \ell_{1 j}$ dependence of the energy $E_{1 j}$ as follows:

$$
\begin{aligned}
E_{1 j}\left(k, \ell_{1 j}\right) \approx & \rho_{0} \frac{\Lambda}{8 \pi}(A \kappa k)^{2} a \ln \left(k \ell_{1 j}+b\right) \times \\
& \frac{\sqrt{1+\pi k \ell_{1 j} / 2}}{2+\Lambda\left(k \ell_{1 j}\right)^{2}} \exp \left(-k \ell_{1 j}\right) .
\end{aligned}
$$

In typical experiments with superfluid ${ }^{3} \mathrm{He}$ and ${ }^{4} \mathrm{He}$ the value of $\Lambda$ varies from 10 to 30 . Therefore we present here parameters $a \approx 1.37, b \approx 1.25$ for $\Lambda=10$ and $a \approx 2.37$, $b \approx 1.17$ for $\Lambda=30$.

c. The third step is to find the relative total crossenergy of all $j \neq 1$ pairs

$$
\begin{aligned}
R(k \ell) & =\sum_{j \neq 1} \frac{E_{1 j}\left(k, \ell_{1 j}\right)}{E_{11}(k)} \\
& \simeq \frac{4 \pi}{(k \ell)^{2}} \int_{\ell_{0}}^{\infty} d \ell_{1 j} \ell_{1 j} \frac{E_{1 j}\left(k, \ell_{1 j}\right)}{E_{11}(k)}
\end{aligned}
$$

which is estimated in Eq. (A7b) in continuous approximation assuming that $j$-lines are randomly distributed around line $i=1$ with mean density $1 / \ell^{2}$ for $\ell_{1 j}$ exceeding, value $\ell$. Generally speaking, the integral (A7b) should contain probability function of vortex separations $\mathcal{P}\left(\ell_{i j}\right)$. Having no reasonable model for it, we choose a simple step function $\mathcal{P}(x)=0$ for $x>1$.

Blending function $g(k \ell)$, defined by Eq. (25), is related to the ratio (A7) as follows:

$$
g(k \ell)=R(k \ell) /[1+R(k \ell)]
$$

Taking oversimplified representation (A6c) of integral (A6a) one computes $g_{0}(k \ell)$, see Eq. (26b):

$$
g_{0}(k \ell)=\left[1+\frac{(k \ell)^{2} \exp (k \ell)}{4 \pi(1+k \ell)}\right]^{-1}
$$

Comparing $\Lambda$-independent function $g_{0}(k \ell)$ with $g_{\text {num }}(k \ell)$, which depends also on $\Lambda$, we have improved its analytical representation by introducing $\Lambda$-dependent rescaling of the argument, see Eq. (26a):

$$
g(k \ell)=g_{0}[0.32 \ln (\Lambda+7.5) k \ell]
$$

The resulting function $g(k \ell)$ gives a very reasonable approximation to the results of "exact" numerical calculation of $g_{\text {num }}(k \ell)$. Therefore in practical calculations we will use analytical form (26) of the blending function $g(k \ell)$.
[1] W. F. Vinen and J. J. Niemela, J. Low Temp. Phys. 128, 167 (2002)

[2] W. F. Vinen, Phil. Trans. R. Soc. A, doi:10.1098/rsta.2008.0084

[3] M. Tsubota, Quantum Turbulence, arXiv:0806.2737

[4] V.B. Eltsov, R. de Graaf, R. Hanninen, M. Krusius, R.E. Solntsev, V.S. L'vov, A.I. Golov, P.M. Walmsley, Turbulent dynamics in rotating helium superfluids Progress in Low Temperature Physics, Vol. XVI, submitted. Also arXiv:0803.3225
[5] S. N. Fisher, A. J. Hale, A. M. Guénault, and G. R. Pickett, Phys. Rev. Lett., 86, 244 (2001)

[6] T. Araki, M. Tsubota, and S. K. Nemirovskii, Phys. Rev. Letts. 89, 145301 (2002)

[7] S.R. Stalp, J.J.Niemela, W.F.Vinen and R.J.Donnely, Physics of Fluids 14, Issue 4, pp. 1377-1379 (2002)

[8] R. Hänninen, R. Blaauwgeers, V. B. Eltsov, A. P. Finne, M. Krusius, E. V. Thuneberg, and G. E. Volovik, Phys. Rev. Lett. 90, 225301 (2003)

[9] A. P. Finne, T. Araki, R. Blaauwgeers, V. B. Eltsov, N. 
B. Kopnin, M. Krusius, L. Skrbek, M. Tsubota, G. E. Volovik, Nature 424, 1022 (2003)

[10] W. F. Vinen, M. Tsubota and A. Mitani, Phys. Rev. Lett. 91, 135301, (2003)

[11] C. Connaughton and S. Nazarenko, Phys. Rev. Lett. 92, 044501 (2004)

[12] D. I. Bradley, D. O. Clubb, S. N. Fisher, A. M. Guénault, R. P. Haley, C. J. Matthews, G. R. Pickett, V. Tsepelin, and K. Zaki, Phys. Rev. Lett. 95, 035302 (2005)

[13] V.B. Eltsov, A. P. Finne, R. Hänninen, J. Kopu, M. Krusius, M. Tsubota, and E. V. Thuneberg, Phys. Rev. Lett. 96, 215302 (2006)

[14] D.I. Bradley, D. O. Clubb, S. N. Fisher, A. M. Guénault, R. P. Haley, C. J. Matthews, G. R. Pickett, V. Tsepelin, and K. Zaki, Phys. Rev. Lett. 96, 035301 (2006)

[15] V.B. Eltsov, A.I. Golov, R. de Graaf, R. Hänninen, M. Krusius, V.S. Lvov, and R.E. Solntsev, Phys. Rev. Letts, 99, 265301 (2007).

[16] P.-E. Roche and C. F. Barenghi, Euro Phys. Lett. 81, 36002 (2008)

[17] R. J. Donnelly, Quantized Vortices in He II (Cambridge University Press, Cambridge, 1991)

[18] Quantized Vortex Dynamics and Superfluid Turbulence, ed. by C. F. Barenghi et al., Lecture Notes in Physics 571 (Springer-Verlag, Berlin, 2001)

[19] V. S. L'vov, S. V. Nazarenko, O. Rudenko, Bottleneck crossover between classical and quantum superfluid turbulence, Phys. Rev. B 76, 024520 (2007).

[20] E.V. Kozik and B.V. Svistunov, Phys. Rev. B 77, 060502(R) (2008)

[21] C. Leith, Phys. Fluids 10, 1409 (1967)

[22] C. Connaughton and S. Nazarenko, Phys. Rev. Lett. 92, 044501 (2004)

[23] E.V. Kozik and B.V. Svistunov, Phys. Rev. Lett. 92, 035301 (2004)

[24] S. Nazarenko, JETP Lett., 84, 585-587 (2006)

[25] P.M.Walmsley, A. I. Golov, H. E.Hall, A.A. Levchenko and W. F.Vinen, Phys. Rev. Letts, 99, 265302 (2007).

[26] It is interesting to make comparison with turbulence of weakly nonlinear waves where the main energy transfer mechanism is due to wavenumber and frequency resonances. In bounded volumes the set of wave modes is discrete and there are much less resonances between them than in the continuous case, so the energy cascades between scales are significantly suppressed. 